\title{
SOLVABLE GROUPS AND MODULAR REPRESENTATION THEORY
}

\author{
BY \\ PAUL FONG( $\left({ }^{1}\right)$
}

In [4] the representation theory of finite solvable groups was studied, and under the assumption of solvability, it was shown that several conjectures of R. Brauer arising from modular representation theory were true. These conjectures are presumably true without the assumption of solvability. In this paper I should like to describe some properties of modular representations of solvable groups which are not shared in common by all finite groups. Solvable groups can be characterized by the existence of Sylow $p$-complements and by the special structure of their principal series; both these features will be exploited. Since one rational prime number $p$ will be fixed for modular representation theory, we shall consider the more general class of $p$-solvable groups, where a group is $p$-solvable if it has a composition series all of whose composition factor groups are either $p$-groups or $p^{\prime}$-groups.

The main results concern the principal indecomposable representations (the indecomposable projective modules in the language of modules). Suppose $(3)$ is a group of order $g=p^{a} g_{0}$, where $\left(p, g_{0}\right)=1$. Let $\Omega$ be a normal algebraic number field containing the $g$ th roots of unity, and $p$ a fixed prime ideal divisor of $p$. The residue class field $\Omega^{*}$ determined by $p$ is then large enough to write the principal absolutely indecomposable representations of $\$$ ). If $\mathfrak{U}$ is such a representation, it is well-known that $p^{a}$ divides the degree $u$ of $\mathfrak{u}$. For a $p$-solvable group, we shall see that $u=p a v$, where $(p, v)=1$ and $v$ is the $p^{\prime}$-part of the degree $f$ of the unique irreducible quotient representation $\mathfrak{F}$ of $\mathfrak{U}$. Moreover, the representation $\mathfrak{U}$ is the induced representation of (5) by a suitable irreducible representation from a Sylow $p$-complement $\mathfrak{S}$ of (5. One consequence of this is that algebraic conjugates of principal indecomposable characters of $\&$ (these are complex-valued functions) are again principal indecomposable characters.

The proofs of the above results are based on the reduction methods of [4]. In $\$ 1$, where the reduction will be briefly described, we shall draw some facts left unformulated in [4]. These are in the nature of relations between the block and group structures of a $p$-solvable group. For example, a question of N. Ito's [7] as to necessary and sufficient conditions when all blocks of a solvable group have full defect is answered. $\$ 2$ contains the results on the principal indecomposable representations.

Received by the editors July 13, 1961. G-9504.

(1) This paper was sponsored in part by the National Science Foundation under grant 
Notation. (\$) is always a finite group of order $g=p^{a} g_{0}$, where $\left(p, g_{0}\right)=1$. For any subset $\mathfrak{A}$ of $\mathfrak{S}, \mathfrak{N}(\mathfrak{A})$ and $\mathfrak{S}(\mathfrak{A})$ are the normalizer and centralizer of $\mathfrak{A}$ in $\mathfrak{S}$. Set $n(\mathfrak{H})=(\mathfrak{N}(\mathfrak{A}): 1)$. If $\mathfrak{A}$ is a one element set $\{A\}$, we write $\mathfrak{N}(A)$ for $\mathfrak{N}(\mathfrak{A})$. An element $G$ of $\mathbb{S}$ is $p$-regular if its order is prime to $p, p$-singular if its order is divisible by $p$. Let $\nu$ be the exponential valuation of the rational field determined by $p$, normalized so that $\nu(p)=1$. The defect $\nu(G)$ of an element $G$ in $\$$ is $\nu(n(G))$; a defect group of $G$ is a Sylow $p$-subgroup of $\mathfrak{N}(G)$.

Let $\Omega$ be a normal algebraic number field containing the $g$ th roots of unity. The condition of normality is not essential, but it simplifies later considerations. The ordinary absolutely irreducible representations of $\&$ can be written with coefficients in $\Omega . \chi_{1}, \chi_{2}, \cdots, \chi_{k}$ will denote the characters of the distinct nonequivalent ones. In $\Omega$ let $p$ be a fixed prime ideal divisor of $p$, with $\mathfrak{o}$ as ring of local integers and $\mathfrak{o} / \mathfrak{p}=\Omega^{*}$ as the residue class field. Denote the image of an element $\alpha$ in $\mathcal{D}$ under the mapping $D \rightarrow \Omega^{*}$ by $\alpha^{*}$. The absolutely irreducible modular representations of $\$$ can be written in $\Omega^{*}$. $\mathfrak{F}_{1}, \mathfrak{F}_{2}, \cdots, \mathfrak{F}_{m}$ will denote the different nonequivalent ones, and $\phi_{1}, \phi_{2}, \cdots$, $\phi_{m}$ their corresponding characters defined with respect to the mapping $0 \rightarrow \Omega^{*}$ (see [1]). The principal indecomposable representations of $\&$ can also be written in $\Omega^{*} . \mathfrak{U}_{1}, \mathfrak{U}_{2}, \cdots, \mathfrak{U}_{m}$ will denote the different nonequivalent ones, and $\Phi_{1}, \Phi_{2}, \cdots, \Phi_{m}$ their corresponding characters, defined also with respect to $\mathfrak{p}$. The arrangement is such that $\mathfrak{F}_{i}$ is the unique irreducible quotient representation of $\mathfrak{u}_{i}$. Unless the word "modular" is used, a character without any other qualifiers will always be one of a complex-valued representation.

If $n$ is a rational integer, it can be factored into two parts, such that one is a power of $p$ and the other is relatively prime to $p$. These we call respectively the $p$-part and the $p^{\prime}$-part of $n$. A $p^{\prime}$-group is a group of order prime to $p$.

1. We assume known the basic notions from modular representation theory as developed in $[1 ; 2]$. Let $\$$ be a $p$-solvable group, and $B_{r}$ a block of (S) with $\mathfrak{D}$ as defect group and $d$ as defect. If $\mathfrak{N}$ is a normal $p^{\prime}$-subgroup of $\mathbb{B}$, a decomposition of the characters in $B_{\tau}$ is induced by $\mathfrak{N}$. The details of this decomposition are essential to our proofs. First $B_{\tau}$ determines a class $\mathcal{C}$ of irreducible characters $\theta_{\rho}$ of $\mathfrak{N}$; namely, the restriction of any (irreducible, irreducible modular, or principal indecomposable) character in $B_{\tau}$ to $\mathfrak{R}$ has the form

$$
m \sum_{\theta_{\rho} \text { in }} e^{\theta_{\rho}}
$$

where $m$ is an integer depending on the character. $\mathcal{C}$ is then a complete class of characters of $\mathfrak{N}$ conjugate in $(S$. By this we mean that given any two characters $\theta_{\rho}$ and $\theta_{\sigma}$ in $\mathcal{C}$, there exists a $G$ in $B$ such that

$$
\theta_{\rho}\left(G^{-1} X G\right)=\theta_{\sigma}(X) \quad \text { for all } X \text { in } \mathfrak{N},
$$

and $\mathfrak{C}$ contains all irreducible characters of $\mathfrak{N}$ related in this manner to a fixed 
character in $\mathfrak{C}$. We shall say that the block $B_{\tau}$ and the class $\mathfrak{C}$ are associated. It may happen that other blocks of $(\mathcal{B}$ are associated to $\mathfrak{C}$; denote the set of all blocks of $\left(\mathfrak{S}\right.$ associated to $\mathcal{C}$ by $\mathfrak{J}$. The block $B_{\tau}$ in $\mathfrak{J}$ has maximal defect among all blocks in $\Im$ if and only if there is a $p$-regular element $V$ in $\mathfrak{N}$ having $D$ as defect group such that $\omega_{\tau}(V) \neq 0$, where $\omega_{\tau}$ is the linear character of the center of the group algebra of $(\xi)$ over $\Omega^{*}$ corresponding to $B_{\tau}$. For any irreducible character $\theta_{\rho}$ of $\mathfrak{N}$, define the inertial group of $\theta_{\rho}$ in $\$$ as

$$
\left\{G \text { in } \$: \theta_{\rho}\left(G^{-1} X G\right)=\theta_{\rho}(X) \text { for all } X \text { in } \mathfrak{N}\right\} \text {. }
$$

Then it is also true that the block $B_{\tau}$ in $J$ has maximal defect among all blocks in $\mathfrak{J}$ if and only if $\mathfrak{D}$ is a Sylow $p$-subgroup of the inertial group of a suitable character in $\mathcal{C}$.

The following is the second step in the decomposition of the characters in $B_{\tau}$. There is some irreducible character $\theta_{\rho}$ in $\mathcal{C}$ such that its inertial group $\mathfrak{I}$ in $\mathbb{S}$ has the following properties: $\mathfrak{I}$ has a block $B_{\tau}^{\prime}$ with $\mathfrak{D}$ as defect group and $d$ as defect. The complete class of characters of $\mathfrak{N}$ conjugate in $\mathfrak{I}$ and associated to $B_{\tau}^{\prime}$ consists of $\theta_{\rho}$ alone. Furthermore, there is a 1-1 correspondence $\chi_{\mu} \leftrightarrow \chi_{\mu}^{\prime}$ between the irreducible characters $\chi_{\mu}$ in $B_{\tau}$ and the irreducible characters $\chi_{\mu}^{\prime}$ in $B_{\tau}^{\prime}$, such that $\chi_{\mu}$ is the character induced by $\chi_{\mu}^{\prime}$. Similarly, there are 1-1 correspondences between the irreducible modular characters $\phi_{\sigma}, \phi_{\sigma}^{\prime}$ of $B_{\tau}, B_{\tau}^{\prime}$, and between the principal indecomposable characters $\Phi_{\sigma}$, $\Phi_{\sigma}^{\prime}$ of $B_{\tau}, B_{\tau}^{\prime}$, such that $\phi_{\sigma}$ is induced by $\phi_{\sigma}^{\prime}$ and $\Phi_{\sigma}$ is induced by $\Phi_{\sigma}^{\prime}$. Finally, $B_{\tau}$ and $B_{\tau}^{\prime}$ have the same matrices of decomposition numbers and the same matrices of Cartan invariants (the matrices being arranged according to the correspondences).

The third step of the decomposition is within the subgroup $\mathfrak{T}$. Because the block $B_{\tau}^{\prime}$ is associated to a one element class $\left\{\theta_{\rho}\right\}$, it determines a factor set $\epsilon$ of $\mathfrak{I}$ in the sense of projective representation theory. $\epsilon$ may be assumed to consist of $s$ th roots of unity, where $(p, s)=1$, but not of $t$ th roots of unity for $t<s$. Every irreducible character $\chi_{\mu}^{\prime}$ in $B_{\tau}^{\prime}$ can be expressed as the product $\chi_{\mu}^{\prime}=\alpha_{\mu} \times \beta$ of two projective characters, where $\alpha_{\mu}$ is the character of an irreducible projective representation of $\mathfrak{T} / \mathfrak{N}$ with factor set $\epsilon$, and $\beta$ is the character of an irreducible projective representation of $\mathfrak{I}$ with factor set $\epsilon^{-1}$, the restriction of $\beta$ to $\mathfrak{N}$ being $\theta_{\rho}$. Similarly, every irreducible modular character $\phi_{\sigma}^{\prime}$ in $B_{\tau}^{\prime}$ can be expressed as the product $\phi_{\sigma}^{\prime}=\gamma_{\sigma} \times \psi$, where $\gamma_{\sigma}$ is the character of an irreducible projective modular representation of $\mathfrak{T} / \mathfrak{N}$ with factor set $\epsilon^{*}$ and $\psi$ is the character of an irreducible projective modular representation of $\mathfrak{T}$ with factor set $\left(\epsilon^{*}\right)^{-1}$, the restriction of $\psi$ to $\mathfrak{R}$ being $\theta_{\rho}$. By a well-known construction of Schur there is a group $\mathfrak{S}$ with a cyclic subgroup $\mathbb{E}$ of order $s$ in its center and $\mathfrak{S} / \mathfrak{F} \simeq \mathfrak{T} / \mathfrak{R}$, having the following properties: each irreducible projective representation of $\mathfrak{T} / \mathfrak{N}$ with factor set $\epsilon$ is induced $\left({ }^{2}\right)$ by an irre-

(2) We have used the word "induced" in two senses so far; the exact meaning will always be clear from context. 
ducible representation of $\mathfrak{S}$; each irreducible projective modular representation of $\mathfrak{T} / \mathfrak{N}$ with factor set $\epsilon^{*}$ is induced by an irreducible modular representation of $\mathfrak{S}$. Let $\chi_{\mu}^{\prime \prime}$ be the character of the representation of $\mathfrak{S}$ inducing the projective representation with character $\alpha_{\mu}$. Similarly, let $\phi_{\sigma}^{\prime \prime}$ be the modular character of the representation of $\mathfrak{S}$ inducing the projective modular representation with character $\gamma_{\sigma}$. SE then has a block $B_{\tau}^{\prime \prime}$ with defect group isomorphic to $\mathfrak{D}$ and defect $d$ such that the irreducible characters in $B_{\tau}^{\prime \prime}$ are precisely the $\chi_{\mu}^{\prime \prime}$ and the irreducible modular characters in $B_{\tau}^{\prime \prime}$ are precisely the $\phi_{\sigma}^{\prime \prime}$. The correspondences $\chi_{\mu}^{\prime} \leftrightarrow \chi_{\mu}^{\prime \prime}$ and $\phi_{\sigma}^{\prime} \leftrightarrow \phi_{\sigma}^{\prime \prime}$ are 1-1, and $B_{\tau}^{\prime}$ and $B_{r}^{\prime \prime}$ have the same matrices of decomposition numbers and the same matrices of Cartan invariants. We shall call $\mathfrak{E}$ the representation group of $B_{\tau}$ ( $\mathfrak{E}$ depends on $\mathfrak{N}) ; \mathfrak{S}$ is a $p$-solvable group if $(\mathbb{S}$ is $p$-solvable.

The above facts are proved in [4] $\$ 2$, except for the assertion that $\Phi_{\sigma}^{\prime}$ induces $\Phi_{\sigma}$, which follows easily, for example, from the fact that $\phi_{\boldsymbol{\sigma}}^{\prime}$ induces $\phi_{\sigma}$ and the Cartan invariants of $B_{\tau}^{\prime}$ and $B_{\tau}$ are the same.

Lemma (1A). Let $\mathbb{H}$ be a p-solvable group and $\mathfrak{N}$ its maximal normal $p^{\prime}-$ subgroup. Let $B_{\tau}$ be a block of $B$ and $C$ the class of irreducible characters of $\mathfrak{N}$ associated to $B_{\tau}$. If $(S)$ is the inertial group of a character in $C$, then the representation group $\mathfrak{S}$ of $B_{\tau}$ has the following structure:

(i) $\mathfrak{S} / \mathbb{E}$ has no normal $p^{\prime}$-subgroups greater than (1).

(ii) $\mathfrak{S}$ has a normal p-subgroup greater than (1).

(iii) Every block of $\mathfrak{S}$ has full defect a.

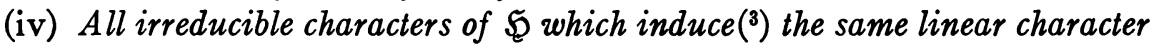
of $\mathbb{E}$ constitute the irreducible characters in a block of $\mathfrak{S}$. There are thus $s$ blocks of $\mathfrak{W}$, where $s=(\mathbb{E}: 1)$.

Proof. Since $\mathfrak{S} / \mathbb{E} \simeq \mathbb{H} / \mathfrak{N}$, (i) is obvious. Let $\mathfrak{R} / \mathfrak{E}$ be the maximal normal p-subgroup of $\mathfrak{E} / \mathbb{E}$. By $[5$, Lemma 1.2 .3$]$, the centralizer of $\mathscr{R} / \mathbb{E}$ in $\mathfrak{E} / \mathbb{E}$ is contained in $\mathbb{R} / \mathbb{E}$. $\mathbb{E}$ has order coprime to $(\mathbb{R}: \mathbb{E})$ so that by a theorem of Schur, $\mathfrak{R}=\mathfrak{D} \mathbb{E}$, where $\mathfrak{D}$ is a $p$-group of order $(\mathbb{R}: \mathbb{E})$ and $\mathfrak{D} \cap \mathbb{E}=(1)$. Since $\mathbb{E}$ is contained in the center of $\mathfrak{F}$, we have in fact $\mathbb{R}=\mathfrak{D} \times \mathfrak{F}$, and therefore $\mathfrak{D}$, being characteristic in $\mathfrak{R}$, is normal in $\mathfrak{W}$. If $V$ is a $p$-regular element in $\mathfrak{E}$ which centralizes $\mathfrak{D}$, then $V \mathbb{E}$ centralizes $\mathscr{L} / \mathbb{E}$ and consequently $V$ belongs to $\mathbb{E}$. If $\mathfrak{B}$ is a defect group of some block of $\mathfrak{S}$, then $\mathfrak{D} \subseteq \mathfrak{P}$. Thus the only $p$-regular elements of $\mathfrak{S}$ centralizing $\mathfrak{B}$ are the elements of $\mathfrak{E}$, and it follows that all blocks of $\mathfrak{S}$ have defect $a$. This proves (ii) and (iii). The proof of (iv) follows from the definition of blocks and $[1,(6 \mathrm{~F})]$. This completes the proof.

An irreducible character $\chi_{\mu}$ of $B$ is defined to be imprimitive if it can be induced from a proper subgroup. Huppert [6, Satz 7] has shown that in a solvable group an irreducible modular character of degree divisible by $p$ is imprimitive. Without the word "modular," this is no longer true as it stands.

(') A third sense of the word "induced." The restriction of an irreducible character of $\mathfrak{W}$ to $\mathbb{E}$ is of course a multiple of a linear character. 
For example, in the split extension of the quaternion group by an automorphism of order 3 , there are irreducible characters of degree 2 which are not induced.

Lemma (1B). Let is be a p-solvable group, and $\chi_{\mu}$ an irreducible character of BS. If $\chi_{\mu}$ is in a p-block of less than full defect $a$, then $\chi_{\mu}$ is imprimitive.

Proof. Let $\chi_{\mu}$ be in the block $B_{r}$. If $\Re$ is the maximal normal $p^{\prime}$-subgroup of $\left(\mathfrak{S}\right.$, and $\mathfrak{C}$ is the class of irreducible characters of $\mathfrak{N}$ which is associated to $B_{\tau}$, then by $(1 \mathrm{~A}) \&$ can be the inertial group of a character in $\mathcal{C}$ only if $B_{r}$ has defect $a$. Therefore by the remarks at the beginning of this section, $\chi_{\mu}$ is imprimitive.

Corollary (1C). Let is be a p-solvable group with abelian Sylow p-subgroups. If $\chi_{\mu}$ is an irreducible character of (s) of degree divisible by $p$, then $\chi_{\mu}$ is imprimitive.

Proof. By $[4,(3 \mathrm{E})] \chi_{\mu}$ must be in a block of less than full defect.

For the rest of this section, we shall use the following notation: If $B$ is a $p$-solvable group, denote by $\mathfrak{N}$ the maximal normal $p^{\prime}$-subgroup of $B$ and by $\mathfrak{B} / \mathfrak{R}$ the maximal normal $p$-subgroup of $\mathbb{S} / \mathfrak{R} .1 \subseteq \mathfrak{N} \subset \mathfrak{P}$ are thus the first three terms of the ascending $p$-series of $(S)$ in the terminology of HallHigman [5].

Lemma (1D). Let (5) be a p-solvable group, $B_{\tau}$ a block of (5), and $\mathbb{C}$ the class of irreducible characters of $\mathfrak{R}$ associated to $B_{r}$. If Bs is the inertial group of a character in $\mathrm{e}$, then $B_{\boldsymbol{r}}$ is the only block of (S) associated to $\mathrm{C}$.

Proof. Let $\mathfrak{G}$ be the representation group of $B_{\tau}$. If $B_{\sigma}$ were another block of $\mathfrak{G}$, different from $B_{\tau}$ but associated to $\mathcal{E}$, then $\mathfrak{E}$ would also be the representation group of $B_{\sigma}$. If $B_{r}^{\prime \prime}$ and $B_{\sigma}^{\prime \prime}$ are then the blocks of $\mathfrak{E}$ corresponding to $B_{\tau}$ and $B_{\sigma}$ respectively, the irreducible characters of $\mathfrak{Q}$ in $B_{r}^{\prime \prime}$ and $B_{\sigma}^{\prime \prime}$ would induce the same linear character of $\mathfrak{E}$, which is impossible by (1A). Therefore $B_{\tau}$ is the only block of (B) associated to $\mathbb{C}$.

THEOREM (1E). Let $1 \subseteq \mathfrak{R} \subset \mathfrak{P}$ be the first three terms of the ascending $p$ series of the p-solvable group $\mathbb{B}$. Let $B_{\tau}$ be a block of $\mathbb{B}$ with defect group $\mathfrak{D}$, and $\mathfrak{e}$ the class of irreducible characters of $\mathfrak{N}$ associated to $B_{r}$. If $\mathfrak{D} \mathfrak{N} \supseteq \mathfrak{P}$, then $B_{r}$ is the only block of $\mathbb{B}$ associated to $\mathfrak{C}$.

Proof. Let $\mathfrak{T}$ be the inertial group of a fixed character $\theta_{\rho}$ in $\mathbb{C}$; we may assume $\mathfrak{T} \supseteq \mathfrak{D}$. If $\mathfrak{T}=\mathfrak{S}$, the result follows from (1D). We may then assume $\mathfrak{T} \subset(B)$. Let $B_{r}^{\prime}$ be the block of $\mathfrak{T}$ which corresponds to $B_{r}$. Since $\mathfrak{T} \supseteq \mathfrak{N}$ and $\mathfrak{T} \supseteq \mathfrak{D}$, it follows that $\mathfrak{T} \supseteq \mathfrak{P}$, and by [5, Lemma 1.2.3, Corollary 2$]$, $\mathfrak{R}$ is also the maximal normal $p^{\prime}$-subgroup of $\mathfrak{T}$. The block $B_{\tau}^{\prime}$ of $\mathfrak{T}$ is associated to the class $\left\{\theta_{\rho}\right\}$ consisting of $\theta_{\rho}$ alone, and thus by (1D) $B_{r}^{\prime}$ is the only block of $\mathfrak{T}$ associated to $\left\{\theta_{\rho}\right\}$. Suppose $B_{\sigma}$ were another block of $(S)$, different from $B_{r}$, associated to $\mathfrak{C}$. Then there would be a block $B_{\boldsymbol{\sigma}}^{\prime}$ of $\mathfrak{T}$ such that the relation 
between $B_{\sigma}$ and $B_{\boldsymbol{\sigma}}^{\prime}$ is exactly like that between $B_{\tau}$ and $B_{\tau}^{\prime}$. But in $\mathfrak{T}, B_{\boldsymbol{\sigma}}^{\prime}$ would be associated to the class $\left\{\theta_{\rho}\right\}$, which is impossible. Therefore $B_{\tau}$ is the only block of $\mathbb{H}$ associated to $\mathfrak{C}$.

CoRollary (1F). Let $\$$ be a p-solvable group, and $B_{\tau}$ a block of full defect $a$. If $\mathfrak{C}$ is the class of irreducible characters of $\mathfrak{N}$ associated to $B_{r}$, then $\mathfrak{C}$ is associated to no other blocks of $B$ other than $B_{r}$.

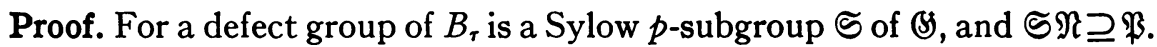

REMARK. The irreducible characters in a block $B_{\tau}$ satisfying the conditions of $(1 \mathrm{E})$ are therefore characterized by their restrictions to $\mathfrak{N}$.

Theorem (1G). Let $\mathbb{B}$ be a p-solvable group and $\mathfrak{N}$ the maximal normal $p^{\prime}$ subgroup of (\$).

(i) Every block of $\$$ is of full defect $a$ if and only if every element in $\mathfrak{N}$ has defect $a$.

(ii) Every block of $\$$ has defect a or defect 0 if and only if every element in $\mathfrak{N}$ has defect $a$ or defect 0 .

Proof. Let $\mathfrak{C}_{1}, \mathfrak{C}_{2}, \cdots, \mathfrak{C}_{r}$ be the distinct classes of irreducible characters of $\mathfrak{N}$ conjugate in $\left(S\right.$, and for $i=1,2, \cdots, r$, let $\theta_{i}$ be a representative of $\mathfrak{C}_{i}$. If among these, $\theta_{1}, \theta_{2}, \cdots, \theta_{t}(t \leqq r)$ are the ones whose inertial groups contain a Sylow $p$-subgroup of $B$, then by $(1 F)$ and the remarks at the beginning of this section, $t$ is also the number of blocks of $B$ of defect $a$. Let $V_{1}, V_{2}, \cdots$, $V$, be representatives of the conjugate classes of $(B)$ contained in $\mathfrak{N} ; r$ is the same number as the number of classes $\mathfrak{C}_{i}$. If $V$ is a $p$-regular element of (s) which centralizes a Sylow $p$-subgroup of $\mathfrak{H}, V$ must belong to $\mathfrak{N}$ by $[5$, Lemma 1.2.3]. Thus all $p$-regular elements of $(\mathcal{B}$ of defect $a$ belong to $\mathfrak{R}$. Since there are $t$ such classes by [2, Theorem 2], we may suppose $V_{1}, V_{2}, \cdots$, $V_{t}$ are the representatives from these classes. Every block of $\$$ has defect $a$ if and only if $r=t$. But then every element in $\mathfrak{N}$ has defect $a$ if and only if $r=t$. This proves (i).

Suppose every element in $\mathfrak{N}$ has defect $a$ or defect 0 , and that there are blocks of $\&$ of defect $d$, where $0 \leqq d<a$. Choose a block $B_{\tau}$ with largest possible defect less than $a$. Then by the remarks at the beginning of this section and $(1 \mathrm{~F})$, a defect group of $B_{\tau}$ must necessarily be a defect group of some element in $\mathfrak{R}$. Thus $B_{r}$ must have defect 0 and every block of $\$$ has defect $a$ or defect 0 . Conversely, suppose every block of $\$$ has defect $a$ or defect 0 . By $[1,(3 \mathrm{C})]$, there exist irreducible characters $\chi_{1}, \chi_{2}, \cdots, \chi_{r}$ of $\&$ such that

$$
\operatorname{det}\left(\chi_{i}\left(V_{j}\right)\right) \not \equiv 0(\bmod \mathfrak{p}) \text {. }
$$

No two of the $\chi_{i}$ have restrictions to $\mathfrak{N}$ which are proportional, and therefore exactly $r-t$ of the characters $\chi_{i}$ belong to blocks of defect 0 . These characters, which we suppose to be $\chi_{t+1}, \cdots, \chi_{r}$, take values divisible by $\mathfrak{p}$ on elements of positive defect $[1,(2.3)]$. In the matrix $\left(\chi_{i}\left(V_{j}\right)\right)$, the submatrix consisting 
of the last $r-t$ rows and the first $t$ columns is the zero matrix modulo $\mathfrak{p}$. Thus from $(1)$ it follows that $\operatorname{det}\left(\chi_{i}\left(V_{j}\right)\right) \not \equiv 0(\bmod p)$ for $i \geqq t+1, j \geqq t+1$, and hence $V_{t+1}, \cdots, V_{r}$ have defect 0 . This proves (ii).

2. If $\mathfrak{U}_{i}$ is a principal indecomposable representation of a group $\mathfrak{H}$, denote its degree by $u_{i}=p^{a} v_{i}$, and the degree of the unique irreducible quotient representation $\mathfrak{F}_{i}$ of $\mathfrak{u}_{i}$ by $f_{i}$.

LEMma (2A). Let $\mathfrak{D}$ be a normal p-subgroup of the group $\mathfrak{B},(\mathfrak{D}: 1)=p^{n}$. If $\overline{\mathfrak{U}}_{i}$ is the principal indecomposable representation of $\overline{\mathfrak{H}}=\mathfrak{H} / \mathfrak{D}$ having $\mathfrak{F}_{i}$ (as a representation of (S) as its irreducible quotient representation, and $\bar{u}_{i}$ is the degree of $\overline{\mathfrak{u}}_{i}$, then $u_{i}=p^{n} \bar{u}_{i}$.

Proof. Let $\Re$ be the regular representation of $\mathfrak{D}$, and $\Im$ the trivial representation of $\mathfrak{D} / \mathfrak{D}$ (over the field $\Omega^{*}$ ). Consider the restrictions $\mathfrak{U}_{i} \mid \mathfrak{D}=m \mathfrak{R}$ and $\overline{\mathfrak{U}}_{i} \mid \mathfrak{D} / \mathfrak{D}=\bar{m} \mathfrak{\Im}$, where $m$ and $\bar{m}$ are certain positive integers. By the Nakayama formulas $[2,(83),(84)] m$ is the multiplicity of $\mathfrak{F}_{i}$ in the representation of $(\mathcal{S}$ induced by $\Im$ (as a representation of $\mathfrak{D}$ ), and $\bar{m}$ is the multiplicity of $\mathfrak{F}_{i}$ (as a representation of $\overline{(S)}$ ) in the representation of $\overline{B S}$ induced by $\Im$. Thus $m=\bar{m}$, and since the degree of $\Re$ is $p^{n}$, it follows that $u_{i}=p^{n} m=p^{n} \bar{u}_{i}$.

THEOREM (2B). Let $\mathfrak{U}_{i}$ be a principal indecomposable representation of the $p$-solvable group (5. If $u_{i}=p^{a} v_{i}$ is the degree of $\mathfrak{U}_{i}$, then $\left(p, v_{i}\right)=1$ and $v_{i}$ is the $p^{\prime}$-part of the degree $f_{i}$ of $\mathfrak{F}_{i}$.

Proof. The proof is by double induction on $a$ and $g=((s: 1)$. We assume that the theorem is true for all $p$-solvable groups having order divisible by at most $p^{a-1}$ and for all $p$-solvable groups of order $p^{a} m$, where $(p, m)=1$ and $p^{a} m<g$.

Let $\mathfrak{U}_{i}$ be in the block $B_{\tau}$ and $\mathfrak{e}$ the class of irreducible characters of $\mathfrak{N}$ associated to $B_{\tau}$, where $\mathfrak{N}$ is the maximal normal $p^{\prime}$-subgroup of $\mathbb{B}$. Suppose $\mathfrak{I}$ is the inertial group of some fixed character in $\mathcal{C}$. If $\mathfrak{T} \subset \mathfrak{S}$, let $B_{\tau}^{\prime}$ be the block of $\mathfrak{T}$ corresponding to $B_{\tau}$, and $\mathfrak{U}_{i}^{\prime}$ and $\mathfrak{F}_{i}^{\prime}$ the principal indecomposable and irreducible representations in $B_{\tau}^{\prime}$ which induce $\mathfrak{U}_{i}$ and $\mathfrak{F}_{i}$ respectively. If $(\mathfrak{T}: 1)=p^{b} r$, where $(p, r)=1$, and $u_{i}^{\prime}$ and $f_{i}^{\prime}$ are the degrees of $\mathfrak{u}_{i}^{\prime}$ and $\mathfrak{F}_{i}^{\prime}$, then by induction, we have $u_{i}^{\prime}=p^{b} v_{i}^{\prime}$, where $v_{i}^{\prime}$ is the $p^{\prime}$-part of $f_{i}^{\prime}$. Since

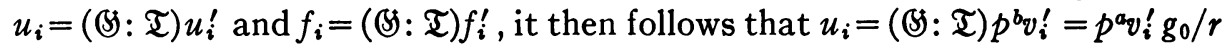
and $v_{i}=v_{i}^{\prime} g_{0} / r$ is the $p^{\prime}$-part of $f_{i}$. We may then suppose $\mathfrak{T}=\mathbb{S}$. Let $\mathfrak{S}$ be the representation group of $B_{\tau}$, and $B_{\tau}^{\prime \prime}$ the block of $\mathfrak{S}$ corresponding to $B_{\tau}$. In $B_{\tau}^{\prime \prime}$ let $\mathfrak{F}_{i}^{\prime \prime}$ be the irreducible modular representation of $\mathfrak{E}$ corresponding to $\mathfrak{F}_{i}$, and $\mathfrak{U}_{i}^{\prime \prime}$ the principal indecomposable representation of $\mathfrak{S}$ having $\mathfrak{F}_{i}^{\prime \prime}$ as quotient representation. By $(1 \mathrm{~A}) \mathfrak{F}$ has a normal $p$-subgroup $\mathfrak{D}$, say of order $p^{n}>1$. It follows then from (2A) that $u_{i}^{\prime \prime}=p^{n} \bar{u}_{i}$, where $u_{i}^{\prime \prime}$ is the degree of $\mathfrak{U}_{i}^{\prime \prime}$ and $\bar{u}_{i}$ is the degree of the principal indecomposable representation of $\mathfrak{S} / \mathfrak{D}$ having $\mathfrak{F}_{i}^{\prime \prime}$ as quotient representation. Since $p^{a} \nmid(\mathfrak{D}: \mathfrak{D})$ we have by induction that $\bar{u}_{i}=p^{a-n} v_{i}^{\prime \prime}$, where $v_{i}^{\prime \prime}$ is the $p^{\prime}$-part of the degree $f_{i}^{\prime \prime}$ of $\mathfrak{F}_{i}^{\prime \prime}$, 
and therefore $u_{i}^{\prime \prime}=p^{a} v_{i}^{\prime \prime}$. But $f_{i}=t f_{i}^{\prime \prime}, u_{i}=t u_{i}^{\prime \prime}$ for some $p^{\prime}$-number $t$, and hence $u_{i}=t u_{i}^{\prime \prime}=p^{a} t v_{i}^{\prime \prime}=p^{a} v_{i}$, since $v_{i}=t v_{i}^{\prime \prime}$. This completes the proof.

Corollary (2C). Let (s) be a p-solvable group of order $g=p^{a} g_{0}$, where $\left(p, g_{0}\right)=1$. For each irreducible modular representation $\mathfrak{F}_{i}$ of $\mathfrak{B}$, let $v_{i}$ be the $p^{\prime}$-part of the degree $f_{i}$ of $\mathfrak{F}_{i}$. If $\mathfrak{M}$ is a Sylow p-complement of $(S)$, then the regular representation of $\mathfrak{M}$ over $\Omega^{*}$ is equivalent to the restriction to $\mathfrak{M}$ of the direct sum $\sum_{i} v_{i} \mathfrak{F}_{i}$. In particular, $g_{0}=\sum_{i} v_{i} f_{i}$.

Proof. The character of the regular representation of $\&$ is $\sum_{i} u_{i} \phi_{i}$ $=p^{a} \sum_{i} v_{i} \phi_{i}$. The restriction of $\sum_{i} v_{i} \phi_{i}$ to $\mathfrak{M}$ is therefore the character of the regular representation of $\mathfrak{M}$, and since $p \nmid(\mathfrak{M}: 1)$, the result follows.

REMARK. (2B) is not true in general. The alternating group on 5 letters has for $p=3$, a principal indecomposable representation of degree 9 whose corresponding irreducible representation has degree 4.

TheORem (2D). Let (S) be a p-solvable group of order $g=p^{a} g_{0}$, where $\left(p, g_{0}\right)$ $=1$. If $\Phi_{\rho}$ is a principal indecomposable character of $\mathbb{B}$, then there exists a $p^{\prime}$-subgroup $\mathfrak{M}$ of $\mathbb{S}$ and an irreducible character $\theta$ of $\mathfrak{M}$ such that $\Phi_{\rho}$ is the character of $\$$ induced by $\theta$.

Proof. The proof is by double induction on $a$ and $g$. We assume that the theorem is true for all $p$-solvable groups having order divisible by at most $p^{a-1}$, and for all $p$-solvable groups of order $p^{a} m$, where $(m, p)=1$ and $p^{a} m<g$. Let $\Phi_{\rho}$ be in the block $B_{\tau}$ of $\mathfrak{B}$ and $\mathcal{C}$ the class of irreducible characters of $\mathfrak{N}$ associated to $B_{\tau}$, where $\mathfrak{N}$ is as usual the maximal normal $p^{\prime}$-subgroup of $\mathbb{H}$. Suppose $\mathfrak{T}$ is the inertial group of some fixed character in $\mathfrak{C}$, and $B_{\boldsymbol{\tau}}^{\prime}$ the block of $\mathfrak{T}$ corresponding to $B_{\tau}$. If $\mathfrak{T} \subset(S)$ and $\Phi_{\rho}^{\prime}$ is the principal indecomposable character in $B_{\tau}^{\prime}$ which induces $\Phi_{\rho}$, then by induction there exists a $p^{\prime}$-subgroup $\mathfrak{M}$ of $\mathfrak{T}$ and an irreducible character $\theta$ of $\mathfrak{M}$ such that $\theta$ induces $\Phi_{\rho}^{\prime}$. Since inducing characters is a transitive operation, it follows that $\Phi_{\rho}$ is the character of $(S)$ induced by $\theta$.

We may then suppose $\mathfrak{I}=\left(\mathfrak{S}\right.$. Let $\mathfrak{S}$ be the representation group of $B_{\tau}$, and $B_{\tau}^{\prime \prime}$ the block of $\mathfrak{S}$ corresponding to $B_{\tau}$. We shall prove the result for the principal indecomposable characters of $\mathfrak{E}$ and show how this implies the theorem for $\left(\mathcal{S}\right.$. By (1A) $\mathfrak{S}$ has a normal $p$-subgroup $\mathfrak{D}$. Let $\mathfrak{F}_{\rho}^{\prime \prime}$ be an irreducible modular representation of $\mathfrak{S}, \phi_{\rho}^{\prime \prime}$ and $\boldsymbol{\phi}_{\rho}$ the characters of $\mathfrak{F}_{\rho}^{\prime \prime}$ as a representation of $\mathfrak{S}$ and $\mathfrak{E} / \mathfrak{D}$ respectively. If $\bar{\Phi}_{\rho}$ is the principal indecomposable character of $\mathfrak{S} / \mathfrak{D}$ corresponding to $\Phi_{\rho}$, then by induction, there exists a $p^{\prime}$-subgroup $\Re / \mathfrak{D}$ of $\mathfrak{S} / \mathfrak{D}$ and an irreducible character $\bar{\theta}$ of $\Re / \mathfrak{D}$ such that $\bar{\theta}$ induces $\bar{\Phi}_{\rho}$. Now $\Re=\subseteq \mathfrak{S}$, where $\mathfrak{S} \simeq \Re / \mathfrak{D}$ and $\mathfrak{S} \cap \mathfrak{D}=(1)$, since $(\mathfrak{D}: 1)$ and $(\Re: \mathfrak{D})$ are coprime. Let $\theta^{\prime \prime}$ be the irreducible character of $\subseteq$ defined by setting $\theta^{\prime \prime}(S)=\bar{\theta}(S \mathfrak{D})$ for $S$ in $\mathfrak{S}$. We claim that $\Phi_{\rho}^{\prime \prime}$ is the character of $\mathfrak{S}$ induced by $\theta^{\prime \prime}$. For if $u^{\prime \prime}=\Phi_{\rho}^{\prime \prime}(1)$, and $\bar{u}=\bar{\Phi}_{\rho}(1)$, then by $(2 \mathrm{~A}), u^{\prime \prime}=(\mathfrak{D}: 1) \bar{u}$ 
$=(\mathfrak{D}: 1)(\mathfrak{D}: \mathfrak{R}) \bar{\theta}(1)=(\mathfrak{W}: \mathfrak{S}) \theta^{\prime \prime}(1)$, so that the degrees check. It will then suffice to check that as a principal indecomposable character of $\mathfrak{S}, \theta^{\prime \prime}$ induces a character of $\mathfrak{S}$ which includes $\Phi_{\rho}^{\prime \prime}$ with multiplicity $m>0$. But this multiplicity $m$, by the Nakayama formulas, is also the multiplicity which $\theta^{\prime \prime}$, as an irreducible modular character of $\mathfrak{S}$, appears in the restriction of $\phi_{\rho}^{\prime \prime}$ to $\mathfrak{S}$. Since the multiplicity of $\theta^{\prime \prime}$ in $\phi_{\rho}^{\prime \prime} \mid \Im$ is the same as the multiplicity of $\bar{\theta}$ in $\Phi_{\rho} \mid \Re / \mathfrak{D}, m$ is positive and therefore $\theta^{\prime \prime}$ induces $\Phi_{\rho}^{\prime \prime}$.

We can now prove the theorem for $\$ B$. By the remarks at the beginning of $\$ 1$, each irreducible modular character $\phi_{\rho}$ in $B_{\tau}$ can be expressed as the product of two projective characters $\phi_{\rho}=\gamma_{\rho} \times \psi$, where $\gamma_{\rho}$ and $\psi$ have factor sets $\epsilon$ and $\epsilon^{-1}$ respectively. Each $\phi_{\rho}^{\prime \prime}$ in $B_{\tau}^{\prime \prime}$ induces the projective character $\gamma_{\rho}$, and since $\Phi_{\rho}^{\prime \prime}=\sum c_{\rho \sigma} \phi_{\sigma}^{\prime \prime}$, where the $c_{\rho \sigma}$ are the Cartan invariants of $B_{r}^{\prime \prime}$, it follows that $\Phi_{\rho}^{\prime \prime}$ induces a projective character of $\mathfrak{S} / \mathbb{E}$ with factor set $\epsilon$. The $p^{\prime}$-subgroup $\mathfrak{S}$ of $\mathfrak{S}$ must contain $\mathfrak{E}$, for if $E$ is in $\mathfrak{E}$, but not in $\mathfrak{S}$, then $\Phi_{\rho}^{\prime \prime}(E)=0$, whereas $\Phi_{\rho}^{\prime \prime}(E)=\zeta u^{\prime \prime}$, for some sth root of unity $\zeta$. Therefore $\theta^{\prime \prime}$

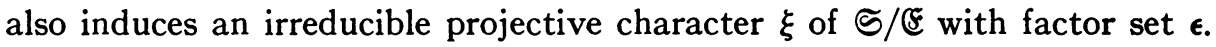
In the given isomorphism $\mathfrak{S} / \mathbb{E} \simeq \mathbb{S} / \mathfrak{N}$, let $\mathfrak{S} / \mathbb{E} \simeq \mathfrak{M} / \mathfrak{N}$. $\mathfrak{M}$ is then a $p^{\prime}$-subgroup of $\mathfrak{S}$. $\xi$ (as a character of $\mathfrak{M}$ ) and $\psi \mid \mathfrak{M}$ are irreducible projective characters of $\mathfrak{M}$ with factor sets $\epsilon$ and $\epsilon^{-1}$ respectively. Define the irreducible character $\theta$ of $\mathfrak{M}$ by the equation $\theta=\xi \times(\psi \mid \mathfrak{M})$. We claim $\Phi_{\rho}$ is induced by $\theta$. For $u_{\rho}=u^{\prime \prime} \times \psi(1)=(\mathfrak{W}: \mathbb{S}) \theta^{\prime \prime}(1) \psi(1)=(\mathfrak{S}: \mathfrak{M}) \xi(1) \psi(1)$, so that the degrees check. As before it will be sufficient by the Nakayama formulas to show that the restriction of $\phi_{\rho}$ to $\mathfrak{M}$ contains $\theta$ with positive multiplicity. Consider first the restriction of $\phi_{\rho}^{\prime \prime}$ to $\mathfrak{S}$. By the Nakayama formulas

$$
\phi_{\rho}^{\prime \prime} \mid \mathfrak{S}=\theta^{\prime \prime}+\sum_{i} a_{i} \theta_{i}^{\prime \prime}
$$

where the irreducible modular characters $\theta_{i}^{\prime r}$ of $\subseteq$ are different from $\theta^{\prime \prime}$. Each $\theta_{i}^{\prime \prime}$ induces an irreducible projective character $\xi_{i}$ of $\mathfrak{S} / \mathfrak{\&}$ with factor set $\epsilon$, and thus

$$
\gamma_{\rho} \mid \mathfrak{S} / \mathfrak{F}=\xi+\sum_{i} a_{i} \xi_{i}
$$

As before it follows that $\theta_{i}=\xi_{i} \times(\psi \mid \mathfrak{M})$ defines an irreducible modular character of $\mathfrak{M}$, and the characters $\theta, \theta_{1}, \theta_{2}, \cdots$ are dist nct by [3, Theorem 5]. Finally then, since $\phi_{\rho}=\gamma_{\rho} \times \psi$,

$$
\begin{aligned}
\phi_{\rho} \mid \mathfrak{M} & =\left(\gamma_{\rho} \mid \mathfrak{M} / \mathfrak{N}\right) \times(\psi \mid \mathfrak{M})=\left(\gamma_{\rho} \mid \mathfrak{S} / \mathfrak{E}\right) \times(\psi \mid \mathfrak{M}) \\
& =\left(\xi+\sum a_{i} \xi_{i}\right) \times(\psi \mid \mathfrak{M})=\theta+\sum a_{i} \theta_{i} .
\end{aligned}
$$

Therefore $\theta$ induces $\Phi_{\rho}$.

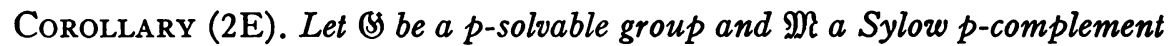
of $\mathfrak{( S )}$. For each principal indecomposable representation $\mathfrak{U}_{i}$ of $\mathfrak{S}$, there exists an irreducible modular representation $\mathfrak{B}_{i}$ of $\mathfrak{M}$ such that $\mathfrak{U}_{i}$ is induced by $\mathfrak{B}_{i}$. 
Proof. If $\Phi_{i}$ is the character of $\mathfrak{U}_{i}$, then there exists a $p^{\prime}$-subgroup $\mathfrak{S}$ of $\mathbb{S}$ and an irreducible character $\theta$ of $\widetilde{S}$ which induces $\Phi_{i}$. Since $p \nmid(\widetilde{S}: 1)$ we may assume by replacing $\subseteq$ by a suitable conjugate that $\subseteq \subset \mathfrak{M}$. If $\mathfrak{Z}_{i}$ is the representation of $\mathfrak{M}$ induced by the representation of $\mathfrak{S}$ whose character is $\theta$, then $\mathfrak{B}_{i}$ is a principal indecomposable representation of $\mathfrak{M}$ which induces $\mathfrak{U}_{i}$. Since $p \nmid(\mathfrak{M}: 1), \mathfrak{B}_{i}$ is in fact irreducible. This completes the proof.

If $\zeta$ is a function of a group $(S)$ with values in $\Omega$, and $\alpha$ is an automorphism of $\Omega$, the algebraic conjugate $\zeta^{\alpha}$ of $\zeta$ under $\alpha$ is defined by $\zeta^{\alpha}(G)=(\zeta(G))^{\alpha}$. If the automorphism $\alpha$ is in the decomposition group of $\mathfrak{p}$, then $\alpha$ will of course permute the principal indecomposable characters of $B$. If $\alpha$ is not in the decomposition group of $\mathfrak{p}$, then this is no longer necessarily true. However, for $p$-solvable groups, we have

TheOREM (2F). Let \& be a p-solvable group and $\mathfrak{A}$ the group of automorphisms of $\Omega$. Then

(i) the algebraic conjugates under $\mathfrak{A}$ of principal indecomposable characters are principal indecomposable characters;

(ii) the algebraic conjugates under $\mathfrak{A}$ of irreducible modular characters are irreducible modular characters.

Proof. Let $\Phi_{1}, \Phi_{2}, \cdots, \Phi_{m}$ be the principal indecomposable characters of (S), arranged so that $\operatorname{deg} \Phi_{1} \leqq \operatorname{deg} \Phi_{2} \leqq \cdots \leqq \operatorname{deg} \Phi_{m}$. We take $\Phi_{1}$ as the character corresponding to the 1-character. $\Phi_{1}$ is then the character of $B$ induced by the 1-character of a Sylow $p$-complement, and thus assumes rational values, so that the theorem is true for $\Phi_{1}$. We apply induction and assume the theorem is true for $\Phi_{1}, \Phi_{2}, \cdots, \Phi_{r-1}$. By (2D) $\Phi_{r}$ is induced by an irreducible modular character $\theta$ of some $p^{\prime}$-subgroup $\mathfrak{M}$. If $\alpha$ is any automorphism in $\mathfrak{A}$, then $\Phi_{r}^{\alpha}$ is induced by $\theta^{\alpha}$. But since $\mathfrak{M}$ is a $p^{\prime}$-group, $\theta^{\alpha}$ is also a principal indecomposable character and thus $\Phi_{r}^{\alpha}$ must be a sum of principal indecomposable characters of $\$$

$$
\Phi_{r}^{\alpha}=\sum_{i} a_{i} \Phi_{i}
$$

where the $a_{i}$ are positive integers. If some $\Phi_{i}$ appearing in (2) has degree equal to $\operatorname{deg} \Phi_{r}$, then we have the case $\Phi_{r}^{\alpha}=\Phi_{i}$ and we are done. If not, then the $\Phi_{i}$ appearing in (2) are necessarily from the set $\left\{\Phi_{1}, \Phi_{2}, \cdots, \Phi_{r-1}\right\}$, and applying $\alpha^{-1}$ to (2), we would have $\Phi_{r}$ expressed as a sum of $\Phi_{1}, \Phi_{2}, \cdots, \Phi_{r-1}$. This is impossible by the linear independence of the $\Phi_{i}$. This proves (i). The proof of (ii) is immediate, for in the vector space over $\Omega$ of functions from the $p$-regular classes of $\&$ into $\Omega$, the subspace generated by $\phi_{1}, \phi_{2}, \cdots, \phi_{m}$ has a unique dual basis under the inner product

$$
\frac{1}{g} \sum f(G) h\left(G^{-1}\right)
$$

namely, $\left\{\Phi_{1}, \Phi_{2}, \cdots, \Phi_{m}\right\},[2,(20)]$. 


\section{REFERENCES}

1. R. Brauer, Zur Darstellungstheorie der Gruppen endlicher Ordnung, Math. Z. 63 (1956), 406-444.

2. R. Brauer and C. Nesbitt, On the modular characters of groups, Ann. of Math. 42 (1941), 556-590.

3. A. H. Clifford, Representations induced in an invariant subgroup, Ann. of Math. 38 (1937), 533-550. 284.

4. P. Fong, On the characters of p-solvable groups, Trans. Amer. Math. Soc. 98 (1961), 263-

5. P. Hall and G. Higman, On the p-length of p-soluble groups, Proc. London Math. Soc. 6 (1956), 1-42.

6. B. Huppert, Lineare auflösbare Gruppen, Math. Z. 67 (1957), 479-518.

7. N. Ito, Some studies on group characters, Nagoya Math. J. 2 (1951), 17-28.

University of Chicago,

Chicago, Illinois

UNIVERSITY OF CALIForNia,

Berkeley, Calmfornia 